\title{
Dynamic Spectrum Access: A New Paradigm of Converting Radio Spectrum Wastage to Wealth
}

\author{
Jide J. Popoola ${ }^{\ddagger}$, Oluwaseun A. Ogunlana, Ferdinad O. Ajie, Olaleye Olakunle, Olufemi A. Akiogbe, \\ Saint M. Ani-Initi, Sunday K. Omotola
}

Department of Electrical and Electronics Engineering, Federal University of Technology, P.M.B. 704, Akure, Ondo State, Nigeria

(jidejulius2001@gmail.com, ayolana2002@yahoo.com, odeyfeddy@yahoo.com, kunvicleye2011@yahoo.com, ayodejiakiogbe@gmail.com, saintzoba@gmail.com, omotolasunday@yahoo.com)

${ }^{\ddagger}$ Corresponding Author: Jide J. Popoola, Department of Electrical and Electronics Engineering, Federal University of Technology, Akure, Ondo State, Nigeria. Tel: +23 4803413/1860, jidejulius2001@gmail.com

Received: 16.08.2016 Accepted: 31.08 .2016

\begin{abstract}
The study presented in this paper reveals the limitations of the current fixed radio spectrum allocation policy as a major bottleneck for availability of radio spectrum for emerging wireless services, devices and applications as a result of its contributions to current radio spectrum artificial scarcity and underutilization problems. In investigating these problems scientifically, series of radio spectrum occupancy studies were carried out in developed nations of the world with little or none in most under-developed nation like Nigeria. In order to ascertain the usage profile of radio spectrum in under-developed nation like Nigeria, actual radio spectrum usage in three different locations in South-West Geo-political zone of Nigeria was carried out. The study was conducted using Aaronia AG HF-6065 V4 spectrum analyzer. The results obtained like other similar studies conducted in other parts of the world show that the usage of radio spectrum varies with time, space and frequency. The results also show that the actual radio spectrum usages in the three locations for the frequency range of 80-2200 $\mathrm{MHz}$ vary from $0.08 \%$ to $64.4 \%$. In addition, the paper enumerates various ways of converting the current wasting spectrum holes to wealth as well as some economic advantages of dynamic spectrum access as a flexible radio access policy that can replace the current fixed radio spectrum allocation policy without compromising the performance of the existing radio being governed by the fixed spectrum allocation policy.
\end{abstract}

Keywords Spectrum holes, spectrum occupancy measurements, dynamic spectrum access, cognitive radio, spectrum trading.

\section{Introduction}

Over the past two decades, the exponential growth in demand for radio or wireless communication services, devices and applications has brought with it the need for a change in the way radio spectrum is being regulated. Currently, the radio spectrum regulatory bodies worldwide are of the opinion that the rigid spectrum management policy granting exclusive right to use licensed spectrum is still efficient. However, observations have shown that the idea of statically apportioning of radio spectrum into blocks and allocated for specific purposes as well as licensed to specific user or operator on either short or long term is long overdue and inefficient. This is because majority of the available radio spectral resources have already been licensed to users indicating that there is little or no room to add any new wireless services, devices and applications unless some of the existing licenses are discontinued [1]. In addition, according to Buddhikot and Ryan [2], current spectrum management also has another serious operational problem as several licensed bands are not efficiently utilized with utilization varying dramatically over frequency, space and time. Furthermore, it was discovered through actual spectral occupancy measurements that vast portions of the licensed radio spectrum are randomly or rarely used by the licensed users [3-10]. For instance, a study conducted by the Federal Communications Commission (FCC) reported by Popoola and Van Olst [11], showed that usage of the licensed radio spectrum varied from $15 \%$ to $85 \%$ in the United State of America (USA). 
Also, as reported by [11], similar actual spectral occupancy measurements showed that as little as $22 \%$ of allocated spectrum is utilized in urban areas and less than $3 \%$ is being utilized in rural areas buttressing the fact that usage of radio spectrum varies with space. Similarly, as reported by [11], observation from actual radio spectrum occupancy measurements conducted in downtown Berkeley showed that allocated frequency bands to licensed users are underutilized especially in the 3-6 MHz, while some other frequency bands are rarely occupied and the remaining frequency bands are heavily occupied showing that radio spectrum usage also vary with frequency. A typical result of these measurements presented in [9] is shown in Fig.1. From these observations, according to [11], the concept of spectrum holes was introduced, which was defined in $[10,12]$ as bands of frequencies assigned to primary users (PUs) also known as licensed users but at a particular time and in specific geographical location the bands are not being utilized by the licensed users.

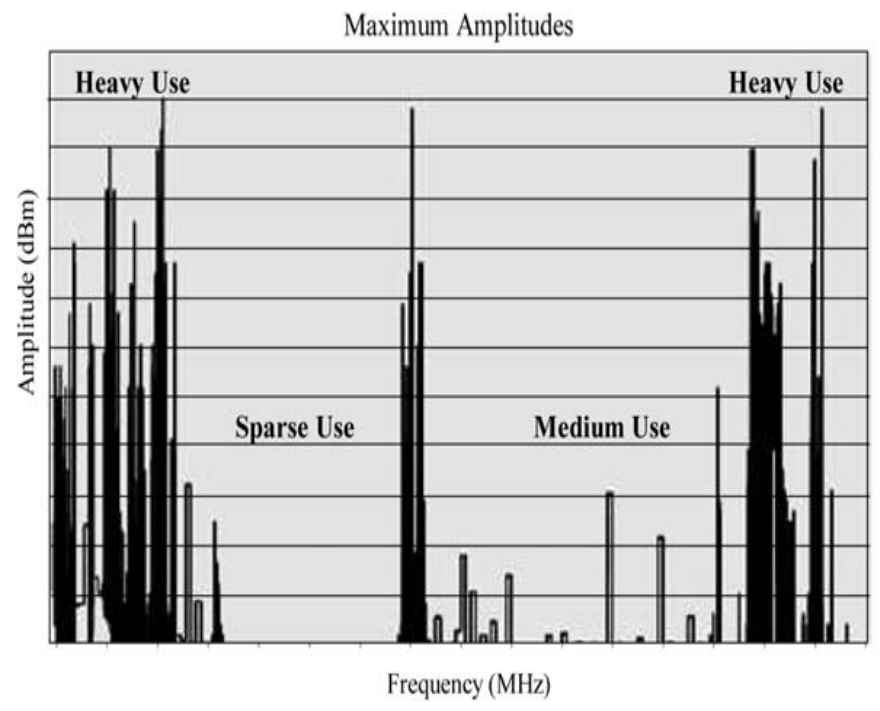

Fig. 1. Typical spectrum under-utilization profile [9]

Generally, according to Najashi and Feng [13], spectrum occupancy measurements have been used to ascertain the level of spectrum utilization by licensed users. The essence of spectrum occupancy measurements is to prove the need for deployment of another radio spectrum access technology for both effective and efficient radio spectrum management. However, out of the several spectrum occupancy measurements conducted all over the world, it was discovered that most were done in USA and Europe with few in Asia and Africa [14]. For instance in South Africa, the spectrum occupancy measurements carried out in the Hatfield area of Pretoria for ultra-high frequency (UHF) band, global system for mobile communications (GSM) 900 $\mathrm{MHz}$ and GSM $1800 \mathrm{MHz}$ bands by [15] show variations in usage of the three bands. The UHF band for instance has an approximately occupancy of $20 \%$ while those of GSM 900 $\mathrm{MHz}$ and GSM $1800 \mathrm{MHz}$ bands are at approximately 92\% and $40 \%$ respectively. Similar study carried out in Kampala, Uganda capital recently using GSM900, GSM1800, the universal mobile telecommunications system 2100 (UMTS2100) and long term evolution 2600 (LTE2600) bands presented in [16] shows variations in those frequency bands considered. The result of the study, gave an average occupancy rates of $8.8 \%$ and $52.4 \%$ respectively for both the uplink and downlink in GSM900 band. The corresponding results for the uplink and downlink occupancy rates for GSM1800 band obtained in [16] are $0.6 \%$ and $13.6 \%$ respectively. Also, the obtained uplink and downlink occupancy results in the study are $0.56 \%$ and $48.7 \%$ respectively for the UMTS2100 band while the corresponding results for the LTE2600 band are $0 \%$ and $0.6 \%$ respectively.

Similarly, in Nigeria, one of the studies on spectrum occupancy measurements in searched literature was conducted and published by [14]. The study presented in [14] was conducted at Gwarinpa District in Abuja. The results obtained by [14] showed that the variation of usage of licensed spectrum in Abuja, the Nigeria Federal Capital Territory, ranges from $17 \%$ to $26 \%$ at $700-2400 \mathrm{MHz}$ frequency bands considered. Furthermore, the results of another recent study on spectrum occupancy measurements [17], which were conducted in Abuja and Katsina both in Nigeria show variations in spectrum usage in the two locations with occupancies variations from $0.45 \%$ to $26 \%$. Thus, in order to ascertain the level of licensed spectrum usage in other parts of the country, the study reported in this paper was embarked upon. It was embarked upon to ascertain whether or not dynamic spectrum access also known as opportunistic spectrum access (OSA) can be proposed in Nigeria as in other nations of the world. The study was conducted in three state capitals (Ado-Ekiti, Akure and Ikeja) in the South-West Geopolitical zone of Nigeria. The full details on how the occupancy measurements were conducted are presented in Section 3 of this paper.

The rest of the paper is organized as follows: In Section 2, a brief background on dynamic spectrum access (DSA) and cognitive radio technology as an enabler of DSA is presented. The details on the field measurements conducted to evaluate the spectrum occupancy in the study areas are presented in Section 3. The results obtained in Section 3 were analyzed and discussed in Section 4. The economic advantages of DSA in converting spectrum holes to wealth by the regulatory bodies and/or licensed users are also presented in this section. Section 5, which is the last section, concludes the paper.

\section{Brief on DSA and Cognitive Radio Technology}

Dynamic spectrum access also known as OSA is defined as a new spectrum sharing paradigm that allows unlicensed or secondary user (SU) to access the idle or unused spectrum otherwise called spectrum holes or white spaces in the licensed spectrum band. It is a flexible radio spectrum access policy to alleviate the current problems of spectrum scarcity and spectrum underutilization in order to increase spectrum utilization [18]. The concept of DSA is to find a means of accessing the unused portion of already assigned licensed spectrum without interfering with the transmission of the PU as illustrated in Fig.2. The type of radio that enables SU to operate in idle portion of the licensed spectrum in this opportunistic manner is known as cognitive radio. 


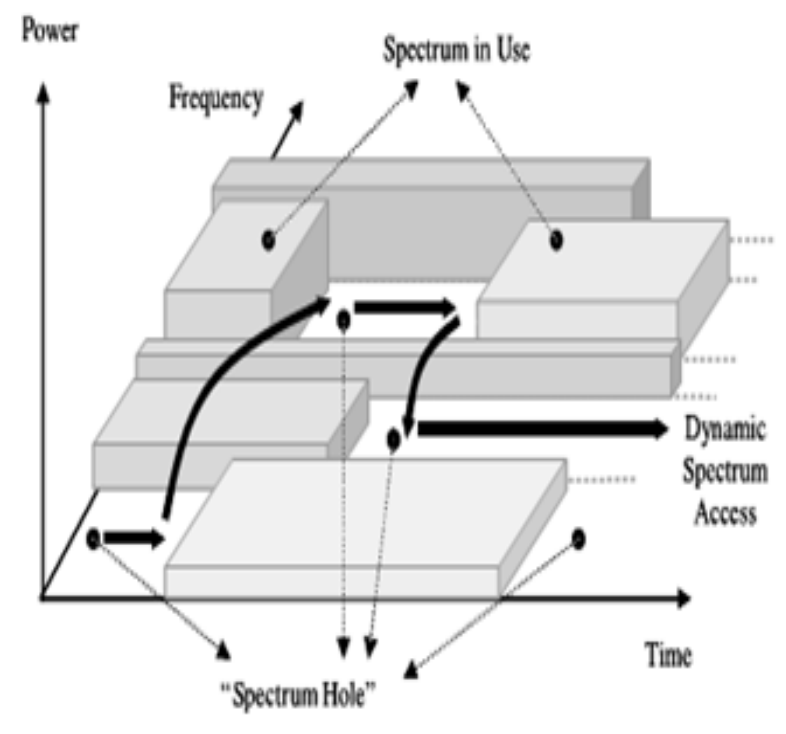

Fig. 2. Spectrum hole concept [9]

Cognitive radio (CR) technology is the main technology that enables already assigned spectrum to be used in a dynamic manner. It is defined as a radio that can change its transmitter parameters based on interaction with the environment in which it operates [9]. The cognitive capability of $\mathrm{CR}$ enables its real time interaction with its environment to determine appropriate communications parameters and adapt to the dynamic radio environment. According to [9], the tasks required for CR to perform this adaptive operation and ensuring interference free communication with the PU of the spectrum is referred to as the cognitive cycle. The three main steps in cognitive cycle as shown in Fig. 3 are spectrum sensing, spectrum analysis and spectrum decision.

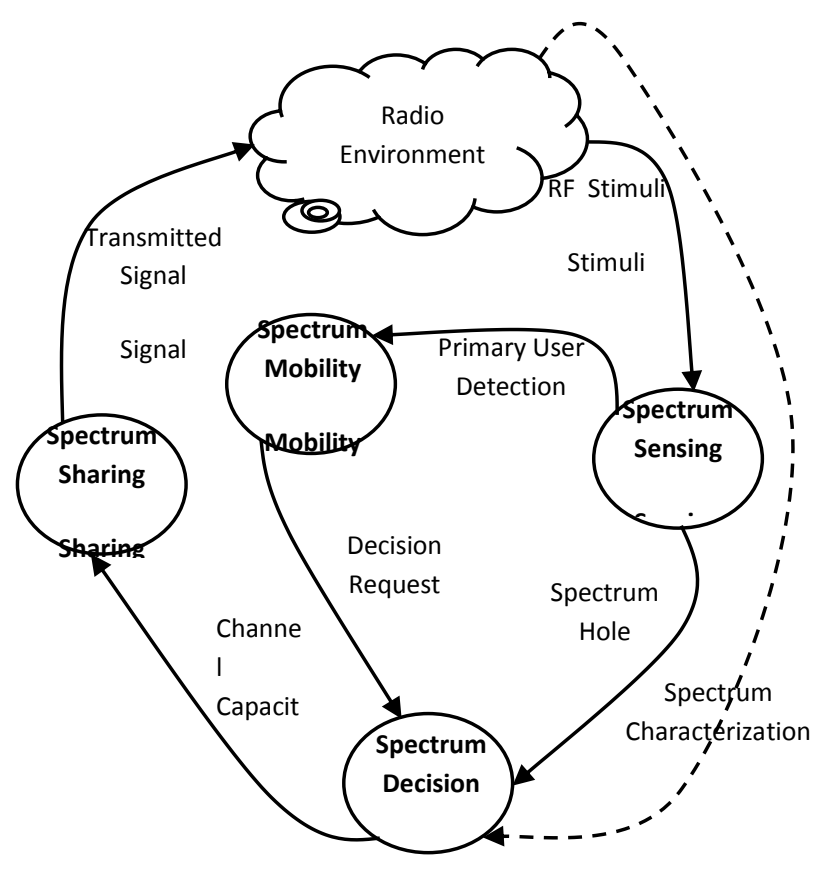

Fig. 3. Cognitive cycle [19]
The process of monitoring the available spectrum band in order to detect the presence of unused spectrum is known as spectrum sensing. Spectrum analysis on the other hand is a means of estimating the characteristics of the detected spectrum holes while spectrum decision is a means of determining the data rates and the transmission mode as well as the bandwidth of transmission with a view to choosing appropriate spectrum band according to spectrum characteristics and user requirements. As availability of spectrum hole is essential for adoption of DSA, the method employed to ascertain the availability of spectrum holes and the level of radio spectrum usage in this study are presented in the next section.

\section{Methodology}

In carrying out the study presented in this paper to ascertain the availability of spectrum holes in Nigeria for proposing adoption of DSA in the country, actual spectrum occupancy measurements were carried out in three states out of the six states that made up of the South-West GeoPolitical zone in Nigeria. The actual spectrum occupancy measurements were conducted in Ado-Ekiti, Akure and Ikeja, which are respectively the state capitals for Ekiti, Ondo and Lagos states respectively. The three locations were were chosen as the good representation of the geo-political zone due to their socio-economic developments, industrial activities, and population size.

In Lagos state, the actual spectrum occupancy measurement was conducted in Ikeja, longitude $3^{0} 20^{\prime} 28^{\prime \prime} E$ and latitude $6^{0} 38^{\prime} 13^{\prime \prime} N$. This study location is a densely populated residential and business area of the state, which is predominantly characterized by schools, banks, office blocks, shopping complex and residential buildings. The location is strategic and ideal for the study because of its proximity to radio and television broadcasting stations, mobile phone base stations, military headquarter, harbour and airport among others radio spectrum users in that vicinity. Likewise, in Akure, the study location lies at longitude $5^{0} 11^{\prime} 42^{\prime \prime} E$ and latitude $7^{0} 15^{\prime} 0^{\prime \prime} N$ while the study location in Ado-Ekiti lies at longitude $5^{0} 13^{\prime} 47^{\prime \prime} E$ and latitude $7^{0} 35^{\prime} 16^{\prime \prime} N$. These study locations are equally strategic locations with proximity to different radio spectrum licensed users. In the three locations, the same measurement setup was employed, which consists of an Aaronia AG HF-6065 V4 spectrum analyzer with a range of $10 \mathrm{MHz}-6 \mathrm{GHz}$, an Aaronia AG HyperLOG antenna, a laptop system that is connected to the spectrum analyzer via a USB cable, and an MCS software specially designed to run on Aaronia AG spectrum analyzers as shown in Fig.4. 


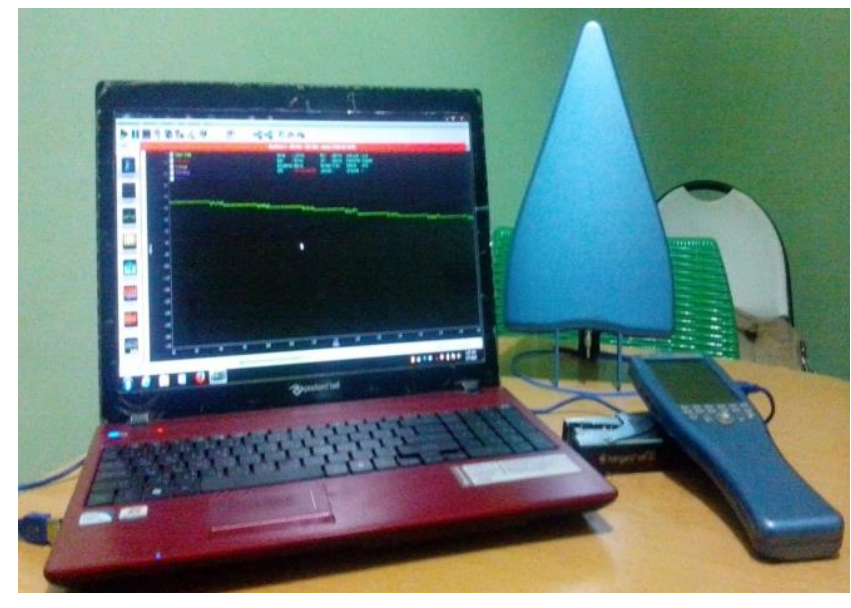

Fig. 4. Measurement set-up

The block diagram representation of the measurement set-up is shown in Fig.5. The arrows in Fig.5 show the direction of flow of signal in the set-up. The antenna receives the signal, the meter quantifies it, and the values of the quantified signals are stored in the laptop which also serves as the display unit for the set-up.

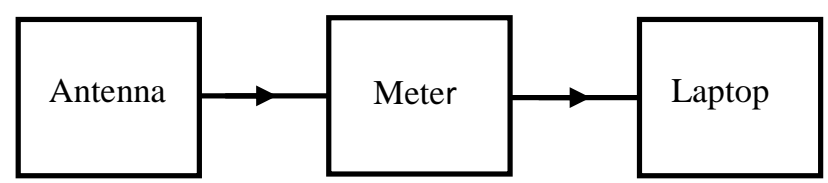

Fig. 5. Block representation of the measurement set-up

In determining the availability of the spectrum hole in any channel, energy detection method was employed, which necessitate the determination of threshold value for each frequency band considered in the three locations. This is because when energy detection method is employed in radio spectrum occupancy measurements, correct determination of the decision threshold upon which a particular channel can be either deemed free or busy is essential since there is no prior-knowledge about the channel. Hence, according to [16], correct setting of the threshold metric is important because high value will lead to under estimation of the channel. On the other hand, if the threshold metric is low it will lead to over estimation of the channel under consideration.

Thus in determining the decision threshold values for each band at each location for this study, the average noise level was first determined by connecting a $50 \Omega$ resistor to the spectrum analyzer [16]. The decision threshold was set by adding $3 \mathrm{~dB}$ to measured thermal noise. The decision threshold obtained varies from one location to another as well as the frequency band since the noise level varies with locations and frequencies. The decision threshold values employed are presented in Table 1. The threshold values were used in determining the actual spectrum occupancy for each frequency band by finding the ratio between the points above the threshold to the total number of points during the measurement period. The actual spectrum occupancy (SO) is defined using the relation;

$$
S O=\frac{N_{0}}{N}
$$

where $N_{0}$ is the total number of points above the threshold value and $N$ is the total number of points during the measurement period. The results obtained from the measurements conducted are presented and discussed in the next section.

\section{Results and Discussions}

Four actual spectral usage profiles for the three locations for $80-150 \mathrm{MHz}, 400-960 \mathrm{MHz}, 900-1300 \mathrm{MHz}$ and $1900-2200 \mathrm{MHz}$ bands out of the eight frequency bands considered are presented graphically in Figs.6-9. The Figures show variations in the spectral usage in those frequency bands in the three locations. Also, the results of the actual spectral usage in the three locations show variations in the spectral usage in time as the occupancy measurements in the three locations were conducted on different days though the same time and days of the week in the same month. The overall results of the study show that the usage of the radio spectrum in the study locations varies with time, location and frequency.

Table 1. Decision threshold for each frequency band

\begin{tabular}{|l|c|l|l|l|l|}
\hline $\begin{array}{c}\text { Frequency band } \\
(\mathbf{M H z})\end{array}$ & $\begin{array}{c}\text { Band } \\
\text { Designation }\end{array}$ & \multicolumn{1}{|c|}{ Applications } & \multicolumn{2}{|c|}{ Threshold value (dBm) } \\
\cline { 4 - 6 } & A & Radio broadcasting band & -65.73 & -55.63 & -54.58 \\
\hline $80-150$ & B & Television and Land Mobile & -53.82 & -72.25 & -72.24 \\
\hline $150-400$ & C & Television broadcasting band & -69.32 & -73.34 & -79.12 \\
\hline $400-960$ & D & Trunk radio services and GSM band & -70.18 & -72.46 & -78.32 \\
\hline $700-1000$ & E & GSM band & -73.11 & -71.22 & -75.55 \\
\hline $900-1300$ & F & Rural Telecoms and GSM bands & -65.66 & -93.43 & -93.13 \\
\hline $1300-1700$ & G & GSM, Oil coy and Satellite broadcast & -80.27 & -84.63 & -86.99 \\
\hline $1700-1900$ & H & 3G band & -86.44 & -86.26 & -94.69 \\
\hline $1900-2200$ & & &
\end{tabular}


INTERNATIONAL JOURNAL Of ENGINEERING TECHNOLOGIES

Jide J. Popoola et al., Vol.2, No.3, 2016

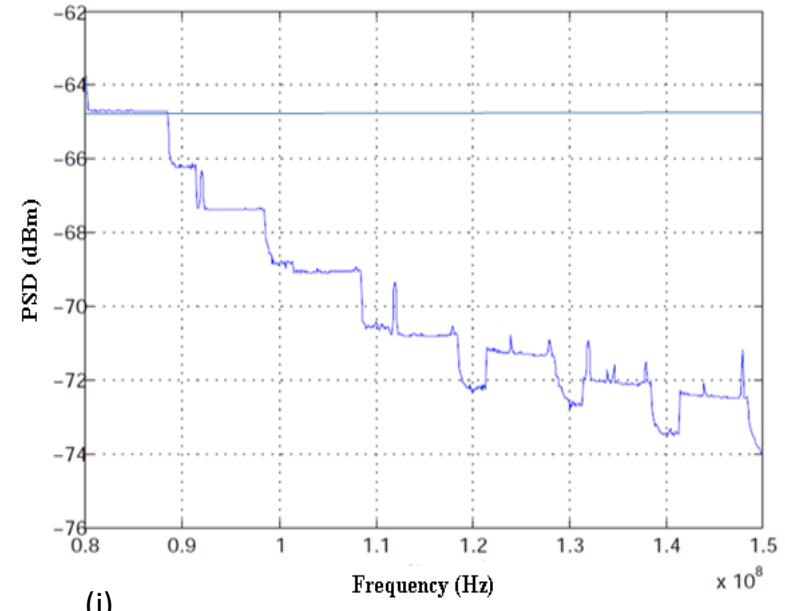

(i)
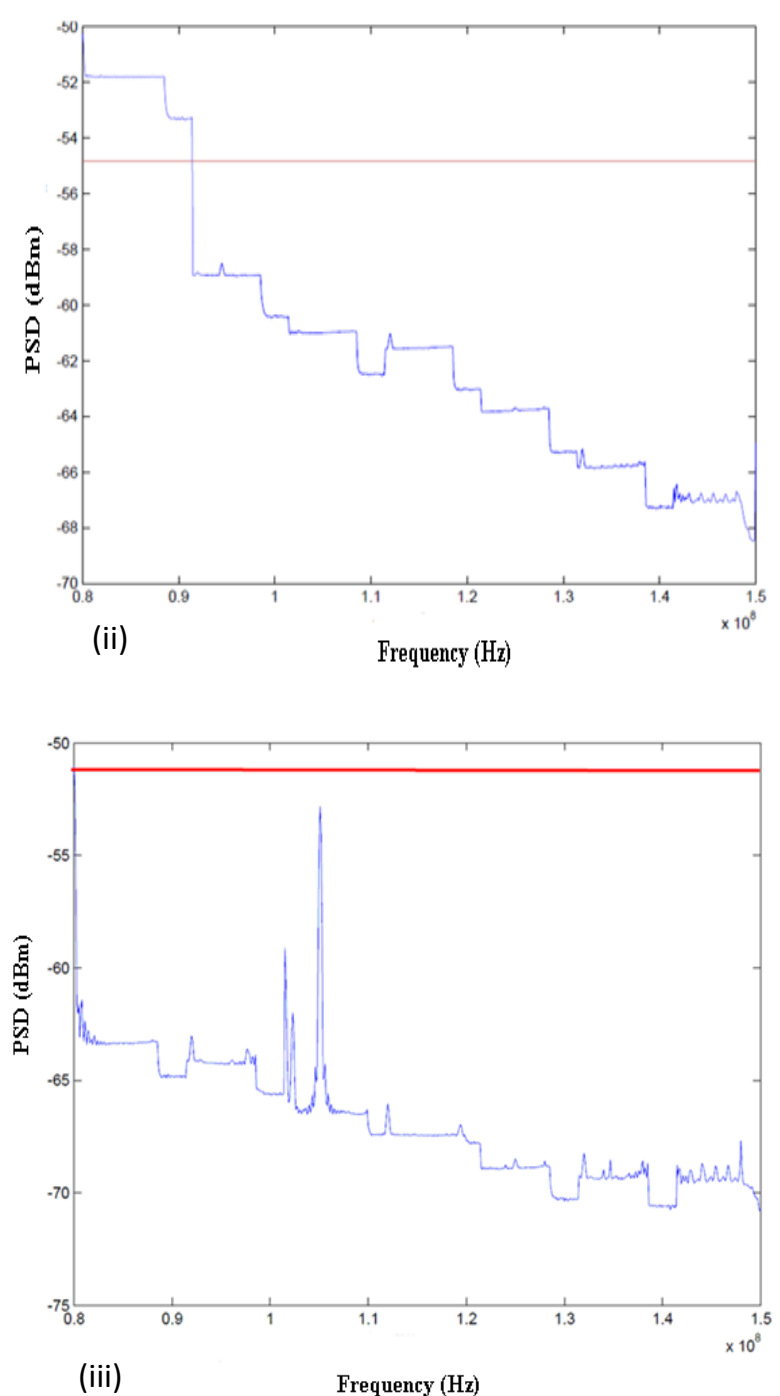

Fig. 6. Spectrum occupancy at 80-150 MHz for (i) AdoEkiti, (ii) Akure and (iii) Ikeja respectively
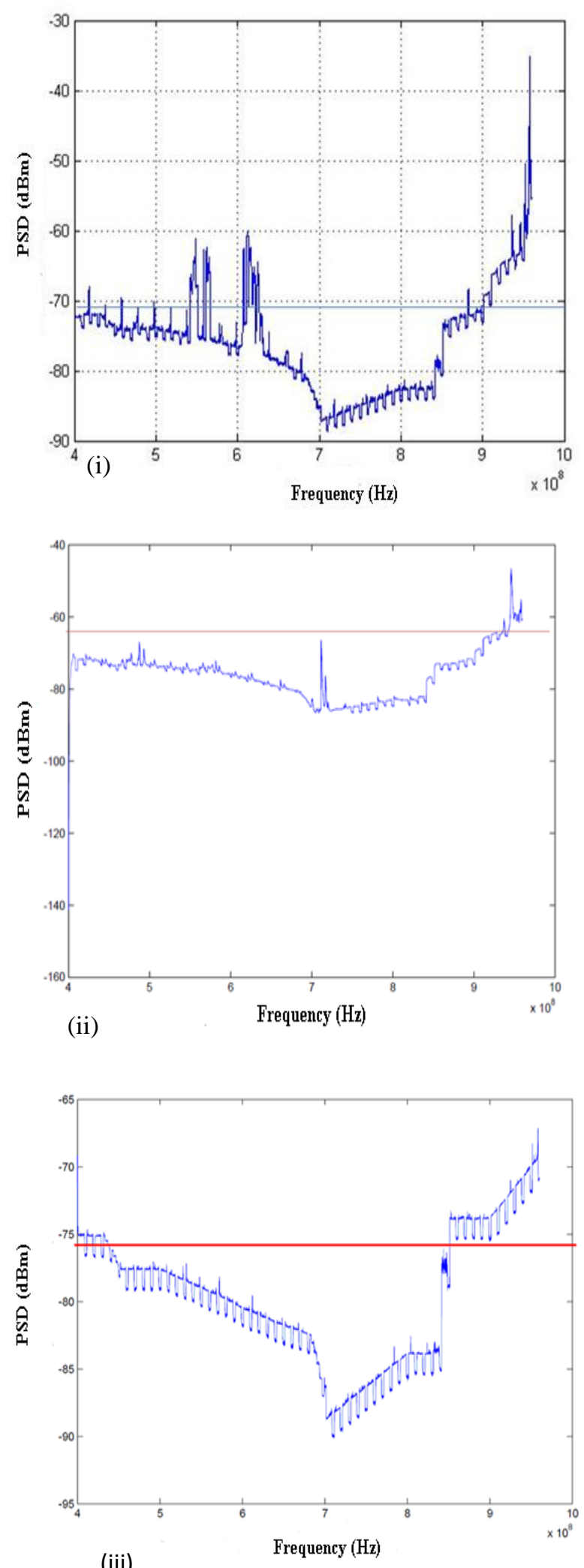

Fig. 7. Spectrum occupancy at 400-960 MHz for (i) Ado-Ekiti, (ii) Akure and (iii) Ikeja respectively 

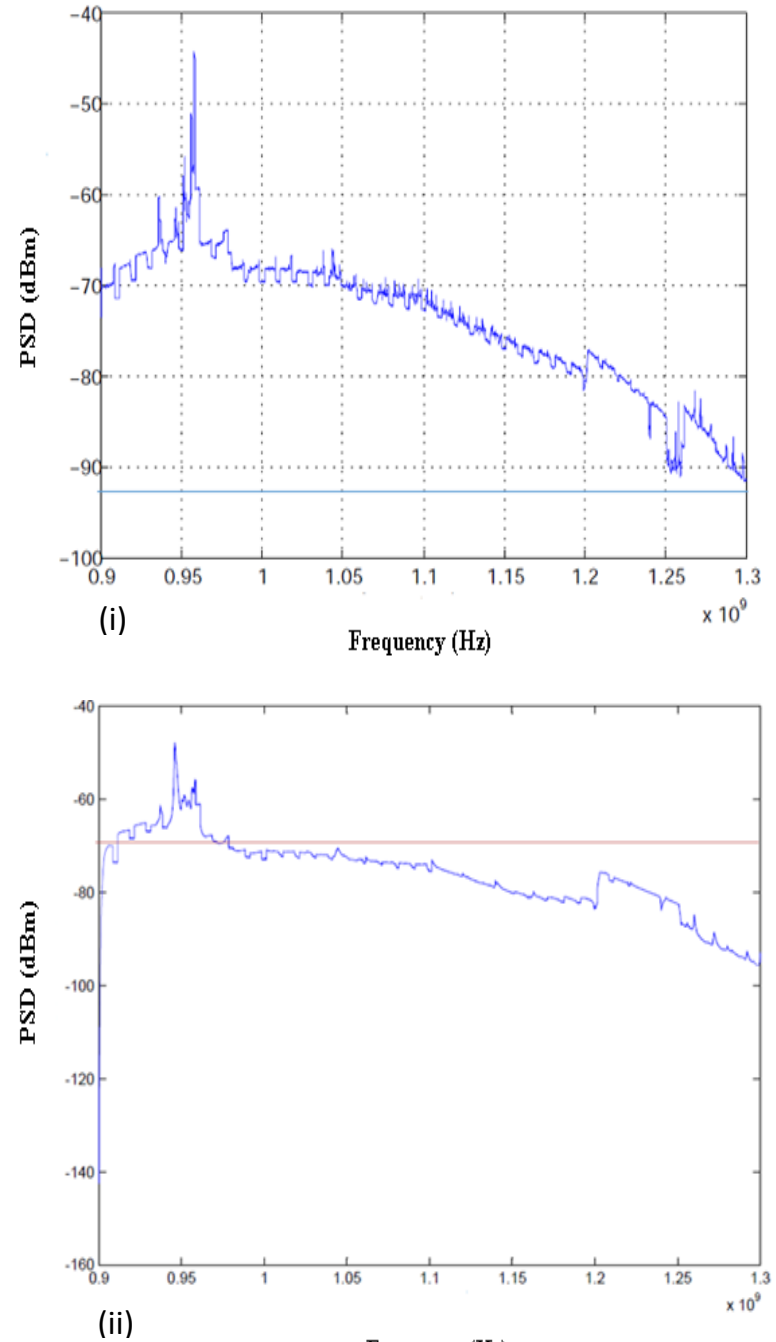

Frequency (Hz)

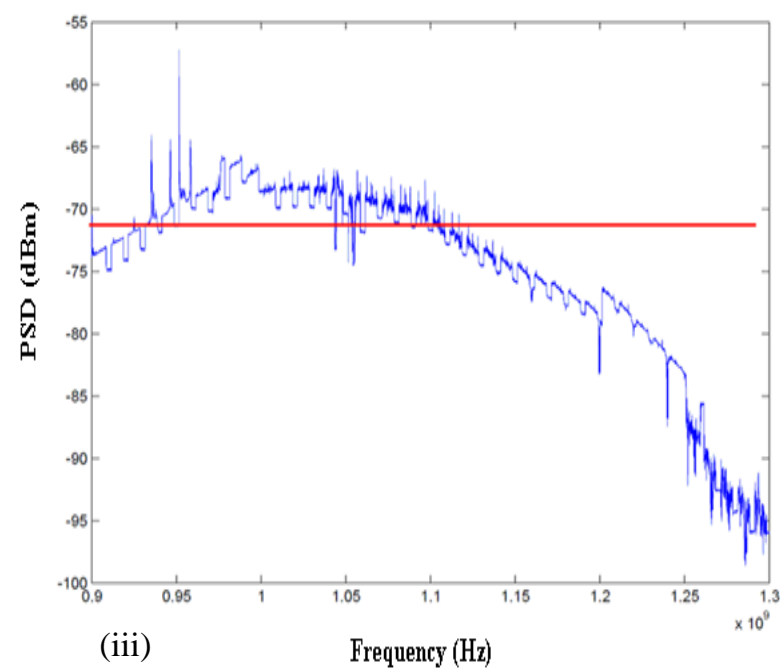

Fig. 8. Spectrum occupancy at $900-1300 \mathrm{MHz}$ for (i) Ado-Ekiti, (ii) Akure and (iii) Ikeja respectively

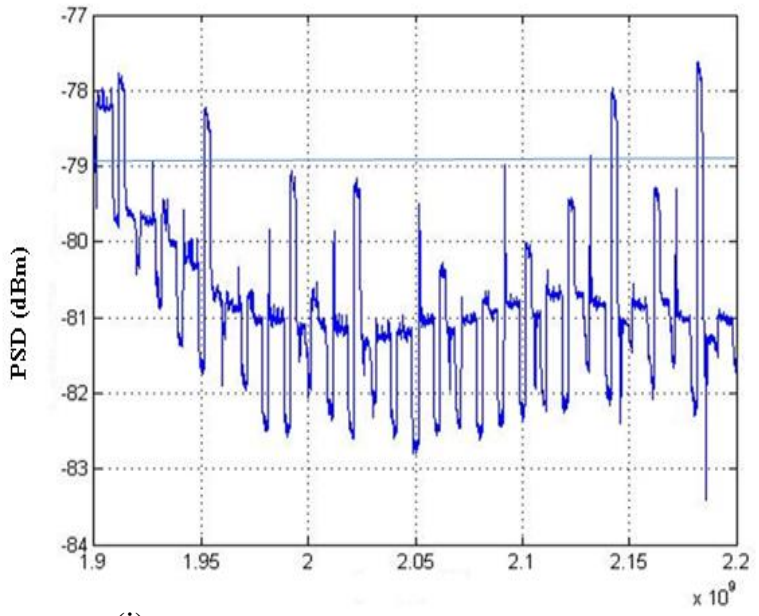

(i)

Frequency $(\mathrm{Hz})$
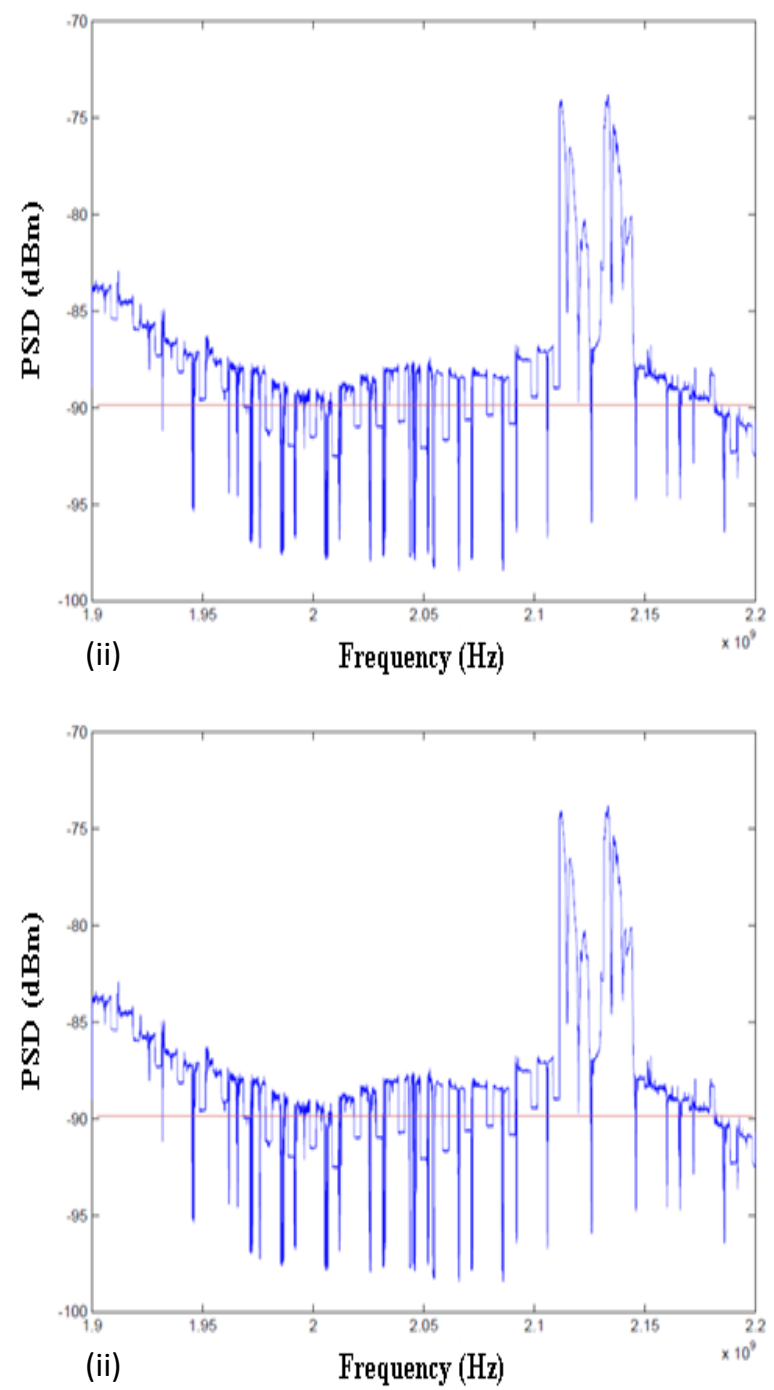

Fig. 9. Spectrum occupancy at 1900-2200 MHz for (i) Ado-Ekiti, (ii) Akure and (iii) Ikeja respectively 


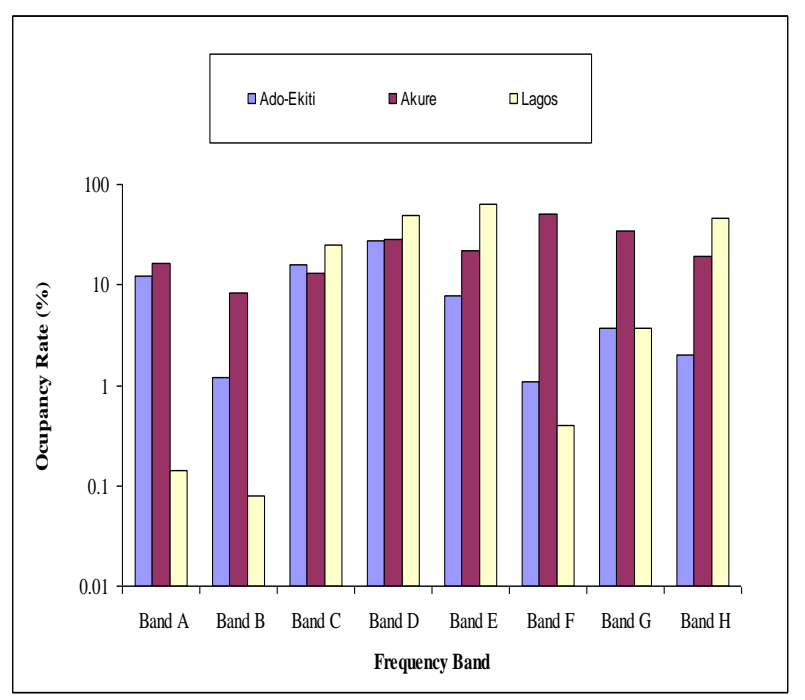

Fig. 10. Overall radio spectrum occupancy for each band

Fig.10 shows the overall radio spectral occupancy for each band using the band designation used in Table 1 . The logarithm scale of the actual occupancy rate obtained was plotted against the frequency band. The logarithm scale was used on the vertical axis in Fig.10 in order to normalise the occupancy rate. The results show variation in the usage of each frequency band with location. The results also show that while a particular frequency was being heavily utilized in a particular location the same frequency was being sparingly utilized in another location. For example, the $80-150 \mathrm{MHz}$ frequency band has utilization level of $12.41 \%$ in Ado-Ekiti while the corresponding values for the frequency band in Akure and Lagos are $16.3 \%$ and $0.14 \%$ respectively. This shows that Band A is sparingly utilised in Ikeja while the band has medium utilization in Akure and Ado-Ekiti. On the other hand, the overall result as shown in Fig.10 shows that Bands D, E and $\mathrm{H}$ which are GSM bands are heavily utilized in Ikeja while the bands are fairly utilised in Akure and sparingly utilised in Ado-Ekiti. Generally, Fig.10 shows that the overall spectrum occupancy in the three locations range from $0.08 \%-64.4 \%$. This shows that there is availability of spectrum holes that can be converted from waste to wealth if DSA is deployed in the country's radio spectrum management. Recently, according to [20], the radio spectrum regulators in the United States and the United Kingdom have given conditional endorsement to DSA mode of spectrum access based on its economic advantages. This implies that adoption of DSA in radio spectrum management policy in underdeveloped nation like Nigeria and generally all over the world has some economic advantages.

One of the economic benefits of DSA is that it enables secondary users of the radio spectrum to utilize the spectrum holes in an opportunistic manner. It thus enhances the efficiency of spectrum usage as well as helping in eradicating the current problem of radio spectrum scarcity and underutilization. Also, the deployment of the DSA technology will enhance the availability of spectral resources for future emerging wireless services, devices and applications [19]. Apart from the fact that DSA can enhance spectral utilization efficiency, another economic advantage of DSA is that it also provides extra revenue for the PU or licensed owner of the spectrum via spectrum trading [19], which according to [21] is the act of selling and buying radio spectrum in a cognitive radio environment. This economic advantage is feasible under DSA because the flexibility involved permits dynamic spectrum market where PUs or licensed owners lease out their spectrum holes or unused channels to generate revenue whenever their channels are not used. Thus the capability of DSA in converting the unused channel that is currently a waste under the current rigid radio spectrum management to wealth for the PUs or licensed owners is a major economic benefit.

In addition, another economic advantage of DSA is that it enhances cost effective access. This enables secondary users to use free and leased spectrum as well as usage of existing radio towers and other infrastructures. According to [19], this act enables smaller scale and lower entry costs become feasible to drive down the service prices. These lower entry costs according to Reference [19] brings about increase in the introduction of new wireless products and services in the marketplace. This cost effective access provides by DSA does not only improve broadband communication system but also promote job opportunity as well as contributing to the nation's economy. Similarly, according to [22], another economic advantage of DSA is that its cost effective access methodology increases faster entry and exist in the market place as well as driving down wireless service prices. This also enhances job creation and socio-economic advancement of the nation.

\section{Conclusion}

Obviously, radio spectrum is a major enabler of wireless communication systems. As a result of its indispensability in wireless communication systems, radio spectrum is being regulated by both the international and national bodies worldwide. However, recent observations have shown that radio spectrum is not effectively and efficiently utilized by licensed owners under the current fixed radio spectrum allocation policy. Specifically, the overall spectrum occupancy or utilization efficiency obtained in this study that ranges from $0.08 \%$ to $64.4 \%$ shows that the current fixed radio spectrum management policy is far overdue and needs to be replaced with flexible management policy that will improve the utilization of radio spectrum. With the rapid progress in DSA technology and economic advantages of DSA, this paper is recommending the replacement of the current fixed spectrum allocation policy with DSA so that the current radio spectrum wastage can be converted to wealth.

Another significant contribution of this study is that it shows the practical relationship of spectrum occupancy with locations. Unlike most similar studies in surveyed literature that consider one or two location(s) with few frequency bands, the study presented in this paper was conducted in three different locations using eight different frequency bands. The results of the study show that spectrum occupancy varies from one location to another. Finally, as a way of concluding this paper, it is obvious that the replacement of the current fixed or rigid radio spectrum management policy with DSA will not only convert the current radio spectrum wastage to wealth and enhancing 
radio spectrum management and its utilization, but will also not compromise the performance of the existing radio being governed by the fixed spectrum allocation policy.

\section{Acknowledgements}

Authors thank Akinlolu Ponnle of Department of Electrical and Electronics Engineering, School of Engineering and Engineering Technology of the Federal University of Technology, Akure, Nigeria for proofreading the manuscript.

\section{References}

[1] I. Budiarjo, H. Nikookar, L.P. Ligthart, (2008) "Cognitive radio modulation techniques", IEEE Signal Pro. Mag., vol. 25, no. 6, pp. 24-34.

[2] M.M. Buddhikot, K. Ryan, (2005) "Spectrum management in coordinated dynamic spectrum access based cellular networks", Proc. of 1st IEEE Int. Conf. on Dynamic Spectrum Access Networks, Baltimore, USA, November 8-11, pp. 299-307.

[3] G. Ding, Q. Wu, Y. Zou, J. Wang, Z. Gao, (2014) “Joint spectrum sensing and transmit power adaptation in interference-aware cognitive radio networks", Trans. on Emerg. Telcomm. Techn., vol. 25, no. 2, pp. 231-238.

[4] J. Wang, G. Diang, Q. Shen, F. Song, (2014) "Spatialtemporal spectrum hole discovery: A hybrid spectrum sensing and geolocation database framework", Chinese Sci. Bulletin, vol. 59, no. 16, pp. 1896-1902.

[5] R. Shukla, D. Sharma, (2013) "Estimation of spectrum holes in cognitive radio using PSD", Int. J. Inf. and Comp Techn., vol. 3, no. 7, pp. 663-670.

[6] A.S. Kadhim, H.M. AISabbagh, (2012) "Detection the spectrum holes in the primary bandwidth of the cognitive radio systems in presence noise and attenuation", Int. J. Comm. Networks and Sys. Sci., vol. 5, no. 10, pp. 685690.

[7] S. Haykin, D.J. Ehomson, J.H. Reed, (2009) "Spectrum sensing for cognitive radio", Proc. of the IEEE, vol.97, no. 5, pp. 849-977.

[8] G. Zhao, J. Ma, G.Y. Li. T. Wu, Y. Kwon, A. Song, C. Yang, (2009) "Spatial spectrum holes for cognitive radio with relay-assisted directional transmission", IEEE Trans. on Wireless Comm., vol. 8, no. 10, pp. 5270-5279.

[9] I.F. Akyildiz, W.Y. Lee, M.C. Vuran, S. Mohanty, (2006) "Next generation/dynamic spectrum access/cognitive radio wireless networks: A survey", Comp. Networks, vol. 50, no. 13, pp. 2127-2159.

[10] S. Haykin, (2005) "Cognitive radio: Brain-empowering wireless communications", IEEE J. on Selected Areas in Comm., vol. 23, no. 2, pp. 201-220.
[11] J.J. Popoola, R. Van Olst, (2011) “Application of neural network for sensing primary radio signals in a cognitive radio environment", Proc. of IEEE AFRICON, Livingstone, Zambia, September 13 - 15, pp. 1-6.

[12] M. Chiani, A. Giorgetti, G. Liva, (2005) "Ultra wide bandwidth communications towards cognitive radio", Proc. Electromag. Comp. EU. Workshop, Rome, Italy, September 19 - 21, pp. 114-117.

[13] B.G. Najashi, W. Feng, (2014) "Cooperative spectrum occupancy based spectrum prediction modelling", $J$. Comp. Infor. Syst., vol. 10, no. 10, pp. 4083-4100.

[14] B.G. Najashi, W. Feng, C. Kadri, (2013) "An insight into spectrum occupancy in Nigeria”, Inter. J. Comp. Sci. Issues, vol. 10, no. 1, pp. 394-399.

[15] S.D. Barnes, P.A.J. van Vuuren, B.T. Maharaj (2013) "Spectrum occupancy investigation: Measurements in South Africa", Measurement, vol. 46, no. 9, pp. 30983112.

[16] G. Ayugi, A. Kisolo, T.W. Ireeta, (2015) "Telecommunication frequency band spectrum occupancy in Kampala Uganda", Inter. J. Research in Eng. and Techn., vol. 4, no. 9, pp. 390-396.

[17] B.G. Najashi, M.D. Almustapha, A.S. Abdi, S.A. Ashurah, (2015) "Spectrum occupancy measurements in Nigeria: Results and analysis,", Int. J. of Comp. Sci. Issues, vol. 12, no. 4, pp. 156-165.

[18] M. Song, C. Xin, Y. Zhao, X. Cheng, (2012) "Dynamic spectrum access: From cognitive radio to network radio", IEEE Wireless Comm., vol. 19, no. 1, pp. 23-29.

[19] J.J. Popoola, R. Van Olst, (2014) "A survey on dynamic spectrum access via cognitive radio: Taxonomy, requirements, and benefits", Universal J. Comm. and Network, vol. 2, no. 4, pp. 70-85.

[20] M. Nekovee, (2010) "A survey of cognitive radio access to TV white spaces", Inter. J. Digital Broadcasting, vol. 2010, pp. 1-11. Online [Available] http//www.hindawi.com/journals/ijdmb/2010 236568. Accessed on May 17, 2016.

[21] K.G. Shin, H. Kitn, A.W. Min, A. Kumar, (2010) "Cognitive radios for dynamic spectrum access: From concepts to reality, IEEE Wireless Comm., vol. 17, no. 6, pp. 64-74.

[22] J.M. Chapin, W.H. Lehr, (2007) "Cognitive radios for dynamic spectrum access- the path to market success for dynamic spectrum access technology, IEEE Comm. Mag., vol. 45, no. 5, pp. 96-102. 